\title{
Malrotation and abdominal pain: A diagnosis eluding the unprepared mind
}

\author{
Rathnaswami Arunachalam ${ }^{1}$
}

Received: 21 November 2015 / Accepted: 22 November 2015/Published online: 10 December 2015

(C) Indian Society of Gastroenterology 2015

Intestinal malrotation (IM) is defined as failure of the midgut to rotate $270^{\circ}$ anti-clockwise in its return from the umbilical cord during fetal development, usually from 5 th to 12 th week. The midgut consisting of a proximal duodenojejunal loop and the distal cecocolic loop, with superior mesenteric artery as its axis, fails in its rotation during development resulting in various types of malrotation. Complete nonrotation and incomplete rotation are the common anomalies encountered in practice. Others are mixed rotation or reverse rotation anomalies. The importance of these various types of anomalies lies in the narrow fixation of the mesenteric base putting the superior mesenteric vascular pedicle at risk of volvulus and abnormally positioned duodenojejunal loop being fixed by peritoneal (Ladd's) bands, resulting in kinking and obstruction of the duodenum. The duodenojejunal loop lies to the right of the vertebra and superior mesenteric artery (SMA) and the entire small bowel in the right half of abdomen. The cecum tends to be mobile or high placed or in the left hypochondrium. There may be associated aplasia of the pancreatic uncinate process.

The majority of patients with IM present, often as an emergency, in infancy $(75 \%)$ or within the first 1 year of life $(90 \%)$ [1]. However a small percentage of them present later in the adult life with nonspecific chronic symptoms which are rarely attributed/recognized to be due to malrotation. Another minority expresses as a chance finding during imaging or surgery for some other cause. Cases do occasionally present due to

Rathnaswami Arunachalam arunarathna@gmail.com

1 Department of Surgical Gastroenterology, SRM Medical College Hospital and Research Centre, SRM Nagar, Potheri, Kattankulathur 603 203, Kancheepuram, Tamil Nadu, India associated anomalies like congenital diaphragmatic hernia [2], esophageal atresia [3], etc.

In infancy and early childhood, IM usually presents acutely, with very little notice, as midgut volvulus which is very often a fatal condition. The child presents with bilious vomiting initially, which is such an important symptom that cannot be passed over. Though bilious vomiting could be due to other causes, in this age group, it is very significant of malrotation and it is considered as a surgical condition unless proved otherwise. Actually, a child younger than 1 year with bilious vomiting may be taken up for surgery, even without upper gastrointestinal (GI) contrast study, as time is very important in preventing intestinal ischemia and the consequent bowel loss. An astute pediatrician and a discerning radiologist with in depth knowledge of malrotation spare no time in arriving at the diagnosis.

Patients with IM do present in adult life with a variety of clinical symptoms like chronic crampy or vague, self-limiting abdominal pain, chronic or recurrent vomiting [4], hematemesis, weight loss, early satiety, constipation, intermittent diarrhea, or occasionally with recurrent acute pancreatitis [5]. IM can lead to intestinal varicosity and can present with GI bleed $[6,7]$. The cause of symptoms may be due to partial, intermittent duodenal obstruction due to kinking, angulation or extrinsic compression by peritoneal bands, or due to partial or recurrent reversible volvulus. The symptoms may also be due to partial and intermittent obstruction to the mesenteric venous and lymphatic system or due to chronic mesenteric arterial insufficiency. As the symptoms are thus often nonspecific, and the condition being less common, the clinician less often attributes this to malrotation. In the article on IM in adults published in this issue of the Journal, the authors, analyzing 64 cases of IM, repeatedly emphasize that patients with chronic, recurrent abdominal pain are often treated erroneously and the diagnosis of malrotation is often missed [8]. 
They regret that even patients with bilious vomiting are referred late to surgery.

During adult life, IM may be an incidental finding during investigation, viz., upper GI contrast study or contrastenhanced computed tomography (CECT) scan [9] for some other disease or discovered incidentally during elective or emergency surgery for a different etiology [10-12]. When suspected, upper GI contrast study is the choice of investigation for diagnosing this condition. The location of the duodenojejunal loop to the right of the midline that is to the right of the spine and in a more anterior location in a lateral view is diagnostic of malrotation. Katz et al. have listed nine criteria for distinguishing normal and abnormal duodenojejunal position on upper GI contrast studies to diagnose malrotation [13]. In the current series, the authors have relied entirely upon the finding of location of duodenojejunal junction for the diagnosis of IM and fail to mention the findings in other investigations which may also be used to make a diagnosis. Significant dilatation of the duodenum may be present in those with duodenal obstruction. The classical finding of entire small bowel on the right side and abnormally placed cecum may not be missed by the insightful radiologist. However, the cecum may be in its normal position or may be mobile and the diagnosis may be missed. On the same score, contrast enema or CECT for colonic position is less reliable for diagnosing IM. Duodenojejunal loop abnormality is seen in $94 \%$ to $97 \%$ of cases in the upper gastrointestinal (UGI) contrast series, but in contrast, only $80 \%$ to $87 \%$ of cases have abnormally positioned cecum on barium enema [14]. Ultrasound (US) and color Doppler are very useful screening tools and they helps in locating the duodenum between the aorta and SMA-aorto mesenteric angle - to exclude IM. Color Doppler imaging showing whirlpool sign is useful in patients with volvulus. These US findings are very useful especially in emergency situation where contrast study of the GI tract may not be practicable.

The superior mesenteric vein (SMV) lying either anterior or to the left of the SMA is a typical finding in malrotation, but however, they are less specific and sensitive. Also, normal position of the SMA and SMV may not rule out malrotation. The "whirlpool" sign, in US and color Doppler study, is typically seen in volvulus of IM with a sensitivity of $82 \%$ to $93 \%$ and specificity of $100 \%$.

The authors reiteratively lament the apathy of treating physicians missing the diagnosis even after performing several investigations like upper GI endoscopy, barium meal series, barium enema, ultrasound, and CECT, often more than once [8]. He stresses that the diagnosis is missed even after diagnostic laparoscopy. This is not unlikely when the imagiologist is not provided with patient's clinical details, the likely possibilities are not indicated or specifically stipulated what to look for (in this case the position of the duodenojejunal loop).
When IM presents as an emergency, and if one has a high index of suspicion, the diagnosis can be made by looking at the position of cecum and small intestine in CT or by ultrasound anomalies of SMV, especially the whirlpool sign. Contrast study in this situation may not be possible. However, the diagnosis will most often be an on table surprise for the surgeon who opens up the abdomen with a diagnosis of intestinal obstruction or infarction. The authors state that the diagnosis in the cases operated as emergency were also made by observing the position of the duodenojejunal junction by the consensus committee. This could only be possible if they have had undergone contrast study before they have developed the acute episode or they were fit enough to undergo the contrast study on presentation.

Ladd's procedure is the choice of operation in IM, wherein the Ladd's bands are sectioned, the base of the mesentery widened, and repositioning of intestine done. Appendicectomy is routinely done to avoid future difficulty in diagnosis $[15,16]$. The procedure can be safely be performed by laparoscopic approach also [17].

All patients diagnosed to have malrotation need to be operated as the surgery is associated with least morbidity and the consequences of not intervening may be life threatening [18]. However, this is a point of debate.

IM in adult life is more often encountered as a chance finding than diagnosed by a clinical perspicacity. This predicament is the subject of poignant interest to the authors who fervently appeal that the scenario be changed. It is very true that this rare entity of IM in adults is often a neglected/missed diagnosis and the patients with vague abdominal symptoms repeatedly visit many clinicians in search of relief, undergo several investigations often repeatedly, and are treated with erroneous diagnosis. Hence, IM should be considered as one of the differential diagnoses in patients with chronic, unexplained, nonspecific abdominal symptoms. It is prudent that stakeholders, endowed with detailed knowledge of this condition, should have a low threshold to envisage, evaluate, and treat this entity.

\section{References}

1. Pumberger W, Kargl S. Malposition of the intestine malposition malrotation volvulus "midgut volvulus". Eur Surg. 2012;44:23747.

2. Salústio R, Nabais C, Paredes B, Sousa FV, Porto E, Fradique C. Association of intestinal malrotation and Bochdalek hernia in an adult: a case report. BMC Res Notes. 2014;7:296.

3. Pachl M, Eaton S, Kiely EM, et al. Esophageal atresia and malrotation: what association? Pediatr Surg Int. 2015;31:181-5.

4. Jadhav RR, Pai VD. Adult intestinal malrotation presenting as recurrent vomiting: a case report. Indian J Surg. 2015;77 Suppl 1: $162-3$. 
5. Sasaki T, Soh H, Kimura T, Hasegawa T, Okada A, Fukuzawa M. Recurrent acute pancreatitis caused by malrotation of the intestine and effective treatment with laparoscopic Ladd's procedure. Pediatr Surg Int. 2005;21:994-6.

6. Palmer OP, Rhee HH, Park WG, Visser BC. Adult intestinal malrotation: when things turn the wrong way. Dig Dis Sci. 2012;57:284-7.

7. Belgaumkar A, Karamchandani D, Peddu P, Schulte KM. Small bowel haemorrhage associated with partial midgut malrotation in a middle-aged man. World J Emerg Surg. 2009;4:1.

8. Fatima H, Shareef HM, Shah A, Satyanarayana G, Prasad GR. Malrotation of midgut in adults, an unsuspected and neglected condition-An analysis of 64 consensus confirmed cases. Indian J Gastroenterol. 2015;34. doi:10.1007/s12664-015-0596-x

9. Zissin R, Rathaus V, Oscadchy A, Kots E, Gayer G, ShapiroFeinberg M. Intestinal malrotation as an incidental finding on CT in adults. Abdom Imaging. 1999;24:550-5.

10. Michalopoulos A, Papadopoulos V, Paramythiotis D, et al. Colonic cancer in a patient with intestinal malrotation: a case report. Tech Coloproctol. 2010;14 Suppl 1:S65-6.

11. Palepu RP, Harmon CM, Goldberg SP, Clements RH. Intestinal malrotation discovered at the time of laparoscopic
Roux-en-Y gastric bypass. J Gastrointest Surg. 2007;11:898902.

12. Ren PT, $\mathrm{Lu} \mathrm{BC}$. Intestinal malrotation associated with colon cancer in an adult: report of a case. Surg Today. 2009;39:624-7.

13. Katz ME, Siegel MJ, Shackelford GD, McAlister WH. The position and mobility of the duodenum in children. AJR Am J Roentgenol. 1987;148:947-51.

14. Applegate KE. Evidence-based diagnosis of malrotation and volvulus. Pediatr Radiol. 2009;39 Suppl 2:S161-3.

15. Nakajima Y, Sakata H, Yamaguchi T, et al. Successful treatment of a 14-year-old patient with intestinal malrotation with laparoscopic Ladd procedure: case report and literature review. World J Emerg Surg. 2013;8:19.

16. Bider K, Kaim A, Wiesner W, Bongartz G. Acute appendicitis in a young adult with midgut malrotation: a case report. Eur Radiol. 2001;11:1171-4.

17. Matzke GM, Dozois EJ, Larson DW, Moir CR. Surgical management of intestinal malrotation in adults: comparative results for open and laparoscopic Ladd procedures. Surg Endosc. 2005;19:1416-9.

18. Fu T, Tong WD, He YJ, Wen YY, Luo DL, Liu BH. Surgical management of intestinal malrotation in adults. World J Surg. 2007;31:1797-803. 